\title{
Performance analysis of peak tracking techniques for fiber Bragg grating interrogation systems
}

\author{
Daniele Tosi, \\ University of Limerick, Optical Fibre Sensors Research Centre, Limerick, Ireland, daniele.tosi@ul.ie \\ Massimo Olivero, Guido Perrone \\ Politecnico di Torino, Department of Electronics, c.so Duca degli Abruzzi, 10129 Torino (TO), Italy, \\ guido.perrone@polito.it
}

\begin{abstract}
In this paper, we propose a spectral correlation-based technique for tracking the wavelength shift of a fiber Bragg grating. We compared this approach, by means of a Monte Carlo numerical simulation, to the typical peak tracking techniques applied in classic interrogation systems. As result, we obtained a considerable gain in terms of noise tolerance (about $20 \mathrm{~dB}$ ), which can be further incremented by selecting large-bandwidth gratings. This permits to increase the power budget of a fiber Bragg grating interrogator without changing the optical layout, overcoming classical limitations of commercial and custom systems. Penalties due to the non-idealities have been evaluated through the same Monte Carlo approach. Finally, we discuss a practical application of the peak tracking techniques to a fiber Bragg grating-based weight sensor, in which we applied the spectral correlation to track both the Bragg wavelength position, spectral deformations due to high strain, and spectral non-linearity.
\end{abstract}

Index Terms - Fiber Bragg grating (FBG), fiber optic sensor (FOS), optical instrumentation, Monte Carlo simulation.

\section{INTRODUCTION}

Fiber Bragg gratings (FBGs) are receiving a considerable interests in optical sensors [1]-[3]: besides owning the advantageous properties of optical technology, the employment of FBGs yields additional benefits such as in-fiber integration, wavelength-encoded operation, possibility to create multiplexed sensor networks (the so-called "smart structures") and predictable reaction to temperature variation. Several interrogation techniques have been documented in literature, using a wide number of principles of operation, such as spectral reconstruction through scanning source [4] or filter [5], matched-filter demodulation [6]-[7], interferometric techniques [8]-[9], birefringence [10]-[11]. Within the last few years, many commercial interrogators have converged to similar optical and processing architectures, most of them based on the straightforward and reliable principle of operation of spectral reconstruction; interrogators measure the wavelength shift of a FBG array simply by scanning a spectral bandwidth with a tunable laser, a tunable filter, or a spectrometer. In this case, a technique for evaluating the peak wavelength from the measured spectrum has to be implemented; typical interrogators define the peak wavelength either as the wavelength correspondent to the spectral 
maximum or as the middle point of the $\mathrm{X}-\mathrm{dB}$ bandwidth (usually $3 \mathrm{~dB}$ for weak gratings and $10 \mathrm{~dB}$ for strong gratings).

A spectral correlation technique, however, permits improving performances of the Bragg wavelength tracking, for all spectrometric and spectral scan-based FBG interrogators, which represent the vast majority of commercial FBG sensing instruments [12]. The correlation method computes the mutual correlation between the reference spectrum and the measured one progressively shifted, and evaluates the FBG wavelength shift as the maximum of the correlation. We performed a Monte Carlo statistical simulation in order to compare the behavior of all peak tracking techniques, using the theoretical expressions of the FBG spectra and reproducing a typical FBG interrogation system, with the purpose of finding the minimum signal-to-noise ratio (SNR) that assesses a correct detection. As result from our simulation, we demonstrate that a FBG interrogator based on spectral reconstruction is capable of gaining a power budget of about $20 \mathrm{~dB}$ only by replacing the peak tracking algorithm, and this margin can be even increased by proper choice of the FBGs. Also, the Monte Carlo technique return quantitative results for FBG design, whereas the optimization of the FBG profile can lead to superior performance in peak tracking.

Finally, peak tracking techniques have been compared in an applicative context of FBG sensor, validating the proposed concept.

\section{THEORY}

The mode-matching technique [13] provides a closed-form expression for the FBG reflectivity that permits to generate grating spectra from the parameters of the refractive index modulation. Hence, the reflectivity of a uniform FBG can be expressed as:

$$
R(\lambda)=\frac{\sinh ^{2}\left(L \sqrt{\kappa^{2}-\hat{\sigma}^{2}}\right)}{\cosh ^{2}\left(L \sqrt{\kappa^{2}-\hat{\sigma}^{2}}\right)-\frac{\hat{\sigma}^{2}}{\kappa^{2}}}
$$

where $R(\lambda)$ is the reflectivity, $L$ is the grating length, $\kappa$ is a parameter that expresses the reflectance per unit length and $\hat{\sigma}$ is a self-coupling coefficient; the parameter $\kappa L$ expresses the strength of the grating. Figure 1 shows typical FBG spectra at $1550 \mathrm{~nm}$ for different values of $\kappa L$ : as the grating strength increases, the maximum reflectivity $R_{\max }=\tanh ^{2}(\kappa L)$ grows and, at the same time, the spectrum enlarges and side lobes become more effective.

When an axial strain $\varepsilon$ is applied to a FBG or its temperature is changed by the quantity $\Delta T$, the resulting spectrum is modified. If the perturbation is low, the Bragg wavelength shift $\Delta \lambda_{B}$ of the FBG can be approximated with a linear relationship [14-15]:

$$
\Delta \lambda_{B}=k_{\varepsilon} \varepsilon+k_{T} \Delta T
$$

where $k_{\varepsilon}$ and $k_{T}$ are the strain- and thermo-optic coefficients; typical values are $k_{\varepsilon}=1 \mathrm{pm} / \mu \varepsilon$ and $k_{T}$ $=10 \mathrm{pm} /{ }^{\circ} \mathrm{C}$ for FBGs operating in the third optical window. For low excitations, the linear model that evaluates the peak wavelength shift can be approximately extended to the whole spectrum: 


$$
R(\lambda, \varepsilon, \Delta T) \cong R\left(\lambda-k_{\varepsilon} \varepsilon-k_{T} \Delta T\right)
$$

Although derived from the ideal theory, Eqs. (1-3) provide a simple analytical model that can be exploited to simulate the behavior of FBGs in optical sensors.

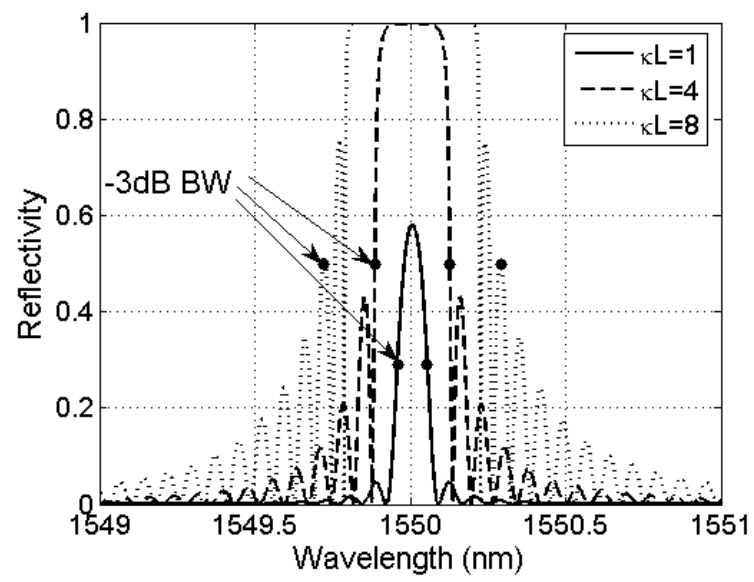

Fig. 1. Theoretical reflection spectra of a $1550-\mathrm{nm}$ FBG with $\kappa L=1$ (solid line), $\kappa L=4$ (dashed line), and $\kappa L=8$ (dotted line).
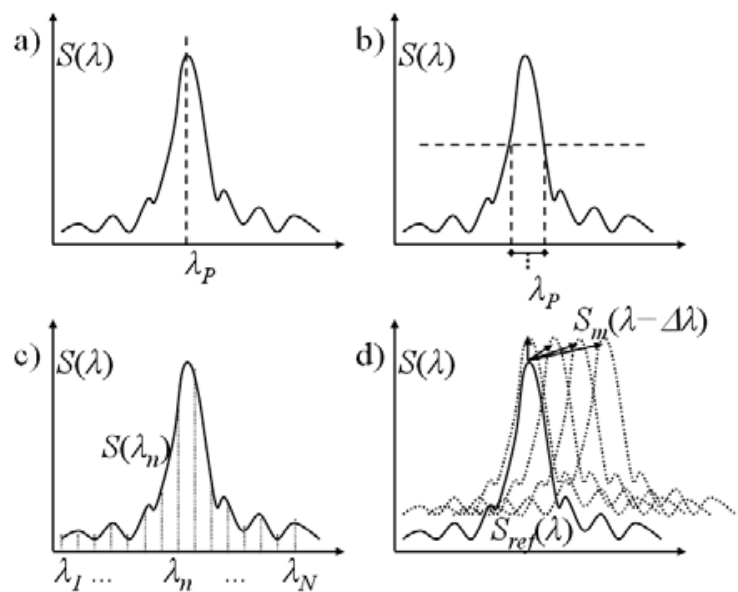

Fig. 2. Peak tracking techniques in FBG spectra: spectral peak (a), bandwidth middle-point (b), centroid (c), spectral correlation (d).

\section{PEAK TRACKING TECHNIQUES}

The typical structure of a FBG static interrogator is based on a spectral reconstruction technique. A tunable laser source, or, alternatively, a broadband source followed by a tunable optical filter, performs a continuous spectral sweep over the wavelength range $\lambda_{1}, \ldots, \lambda_{N}$, with sampling resolution $\delta \lambda$. The source is coupled to the FBG array, and the backreflected power is received with a photodetector, synchronized to the spectral sweep. Hence, the whole FBG spectrum over the interrogator bandwidth is reconstructed.

With this arrangement, a technique for extracting the peak position, and consequently the spectral shift, of each FBG has to be defined [16-18]. The typical tracking method is to estimate the peak wavelength as the maximum of the spectral response or, more commonly, to define the FBG pitch as the middle-point of the $\mathrm{X}-\mathrm{dB}$ bandwidth. A possible alternative approach is to estimate the peak position as the centroid of the FBG spectrum $S(\lambda)$ : 


$$
\lambda_{C}=\frac{\sum_{n=1}^{N} \lambda_{N} S\left(\lambda_{N}\right)}{\sum_{n=1}^{N} S_{N}}
$$

The alternative technique proposed in this paper, and illustrated in Fig. 2, is based on the exploitation of the linear strain/temperature-spectrum relationship. The FBG spectrum is measured, with the same setup, at the initial time, obtaining the reference spectrum $S_{\text {ref }}(\lambda)$. Then, at each instant the measured spectrum $S_{m}(\lambda)$ is compared to the reference one, and the wavelength shift $\Delta \lambda_{s}$ is estimated as the maximum of the correlation between the reference spectrum and the shifted replica of the measured one [16]:

$$
\Delta \lambda_{s}=\left.\Delta \lambda\right|_{\substack{\max \\ \Delta \lambda}}\left(S_{\text {ref }}(\lambda) S_{m}(\lambda-\Delta \lambda)\right)
$$

\section{SIMULATION}

\section{A. Outline}

The core of the paper relies on the proposed benchmark for peak tracking evaluation, based on Monte Carlo simulation [19]. The Monte Carlo approach allows simulating each peak tracking technique in presence of arbitrary SNR, and with any FBG profile, while traditional simulation techniques operate only with a single FBG profile [16-17]. Thus, the use of Monte Carlo returns not only a qualitative estimate of the superior performance of correlation-based tracking, but also crafts specific FBG design considerations for maximizing interrogation performance.

Thus, the purpose of our simulation is to evaluate the noise tolerance of each peak tracking technique, assuming the typical parameters of a FBG interrogator (either based on scanning filter or spectrometer) and the theoretical behavior of grating spectra. We apply a statistical simulation derived from the Monte Carlo technique: we generate several instances of additive noise, multiplied for different values of SNR, and then evaluate the error on the peak estimation. The goal is to determine, for each value of the grating strength $\kappa L$, the threshold SNR, defined as:

$$
S N R_{T H}=\left.S N R\right|_{P\left(\left|\Delta \lambda_{e r r}\right|\left\langle\Delta \lambda_{T H}\right)=P 0\right.}
$$

where $\mathrm{P}\left(\left|\Delta \lambda_{e r r}\right|<\Delta \lambda_{T H}\right)$ is the probability of correct detection, i.e. the error $\Delta \lambda_{e r r}$ between the FBG wavelength $\lambda_{P}$ and the estimated peak $\lambda_{P e}$ is lower than the acceptable error $\Delta \lambda_{T H}$, and $P 0$ is the target probability. We define the SNR as:

$$
S N R=\frac{R_{\max }}{\sigma_{n}^{2}}
$$

where $\sigma_{n}{ }^{2}$ is the noise variance; this assumption permits to evaluate the SNR related to the FBG reflectivity. As known from the theory of statistical simulations, the Monte Carlo approach does not assess that the error is limited, unless the noise probability density is limited, but gives information only on the probability of correctness of detection. 


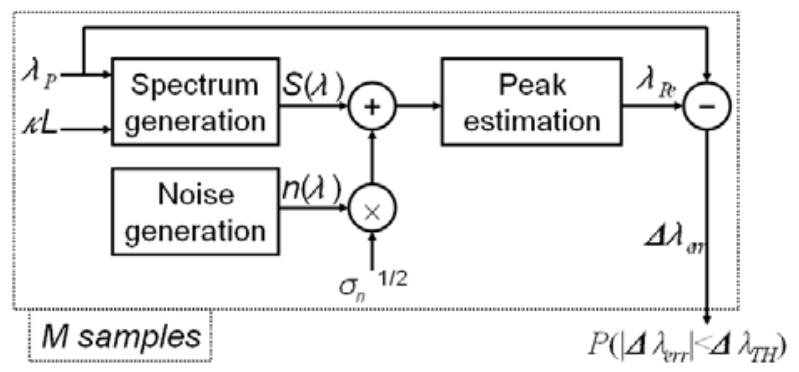

Fig. 3. Schematic of the Monte Carlo simulation approach.

The operative approach of the simulation is schematized in Figure 3. As first step, the reflection spectrum of a FBG with peak wavelength $\lambda_{P}=1550 \mathrm{~nm}$ and a fixed value of $\kappa L$ is generated, according to (1); the spectrum is evaluated over the wavelength range 1547-1553 nm, with step $\delta \lambda=1$ pm. The sampling grid is consistent with the typical commercial FBG interrogator [20-21]. Then, for each Monte Carlo iteration, a noise instance with unitary variance and a fixed probability density function is generated, normalized to the desired SNR value and summed to the FBG spectrum. Since we assume, from the linear model described by (3), that the spectrum shifts without distortion, there is no need to include a wavelength shift in the simulation. Then, the FBG peak is estimated with all the previously described techniques, obtaining the error $\Delta \lambda_{e r r}$; we set the error threshold for correct peak detection $\mathcal{A}_{T H}$ as $10 \mathrm{pm}$, which is close to the typical accuracy of a FBG interrogator. The simulation is repeated $M=1000$ times, setting the target probability of correct detection $P 0=96 \%$ and changing the SNR, in order to evaluate $S N R_{T H}$ for each value of $\kappa L$.

Since the peak tracking performances depend on the noise distribution, we performed the Monte Carlo simulation with both gaussian and uniformly distributed noise. These almost uncorrelated noise profiles tend to advantage the spectral correlation technique; however, realistic systems include a whitening filter that removes the residual correlation bringing the system back to an almost uncorrelated noise shape.

\section{B. Results and discussion}

The results of Montecarlo simulation, obtained with additive white gaussian noise, are reported in Figure 4. The graph clearly shows the completely different behavior of the peak tracking approaches, with a strong dependence on the FBG bandwidth.

The pitch wavelength estimated through the peak of the spectral response exhibits the worst performances: the minimum required SNR is $34.2 \mathrm{~dB}$ in the best case $(\kappa L=0.4)$, but performances strongly decrease when the FBG bandwidth grows, overcoming $100 \mathrm{~dB}$ for $\kappa L>5$. Clearly, this technique has unacceptable performances unless the FBG is extremely selective. The peak estimation based on the -3dB bandwidth yields much better results: the target SNR lays on a floor (19.4 $\mathrm{dB}-$ $24.4 \mathrm{~dB}$ ) for $\kappa L$ ranging from 0.4 to 3, hence for $14.4 \%$ - 99\% reflectivity, and tends to decrease for larger FBGs. The non-monotonic increase of $S N R_{T H}$ for high values of $\kappa L$ can be explained by the 
effect of spectral side-lobes, which are progressively included in the -3dB bandwidth and are more selective than the main lobe. The centroid algorithm, instead, exhibits the opposite trend: the threshold SNR monotonically decreases with $\kappa L$, requiring a strong SNR for poorly reflective FBGs, but it is convenient with respect to the $-3 \mathrm{~dB}$ bandwidth (BW) tracking for $\kappa L>9\left(S N R_{T H} \approx 32 \mathrm{~dB}\right)$. Finally, the spectral correlation is by far the most efficient technique, since it requires the lowest SNR for each value of $\kappa L$. The $S N R_{T H}$ gain with respect to the bandwidth tracking is significant: for $\kappa L=1.8$, which is the best case of the -3dB BW curve, the SNR required by the spectral correlation techniques is 18.2 $\mathrm{dB}$ lower. For $\kappa L>2.5, \mathrm{SNR}_{\mathrm{TH}}$ is lower than $0 \mathrm{~dB}$, which means that the system is extremely noisetolerant; for $\kappa L=12$, the target SNR for the spectral correlation is $-5.3 \mathrm{~dB}$, with a difference of $34.0 \mathrm{~dB}$ - $34.9 \mathrm{~dB}$ with respect to $-3 \mathrm{~dB} \mathrm{BW}$ and centroid curves, respectively, at the same $\kappa L$. As expected, the spectral enlargement occurring when $\kappa L$ increases permits a better alignment of the spectra. The case $\kappa L=0.1\left(R_{\max }=1 \%\right)$, reported for completeness, is not of practical interest.

The variation of the noise probability density from gaussian to uniform distribution does not modify the behavior of the $S N R_{T H}(\kappa L)$ curves, as shown in Figure 5, although performances are slightly improved. Peak and -3dB BW curves reproduce the same trend of gaussian noise, but the target probability is achieved with a lower SNR; the gain, with respect to the gaussian probability density, is $7.3 \mathrm{~dB}-14.1 \mathrm{~dB}$ for the peak curve and $3.0 \mathrm{~dB}-6.5 \mathrm{~dB}$ for the $-3 \mathrm{~dB}$ BW tracking, with a small dependence on $\kappa L$. On the other hand, centroid and correlation curves exhibit the same behavior of Figure 4 , with a slight $( \pm 1.5 \mathrm{~dB})$ difference that can be possibly attributed to the low number of Monte Carlo samplings.

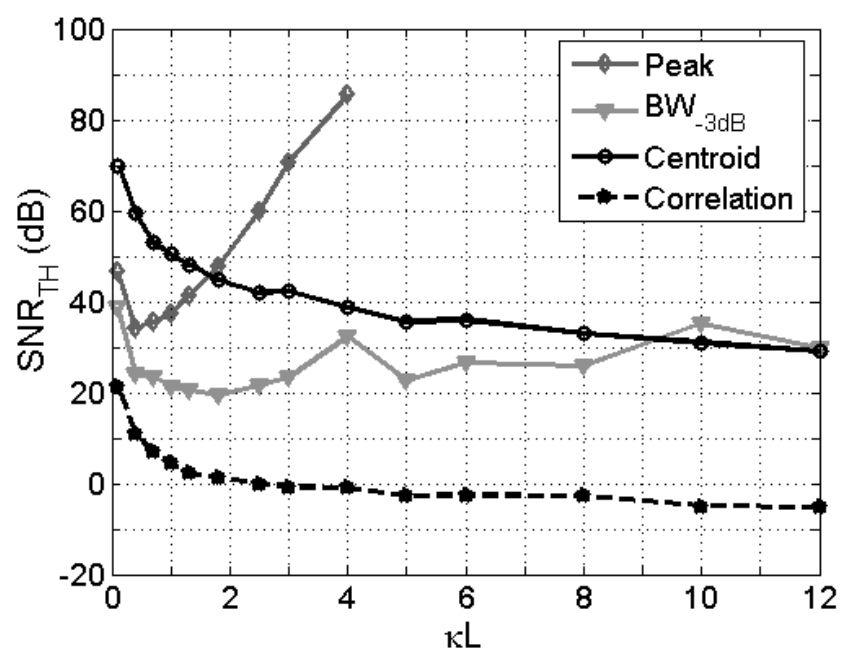

Fig. 4. Evaluation of the target SNR as a function of $\kappa \mathrm{L}$ for each tracking technique, performed with a 1000-sample Monte Carlo simulation with gaussian noise. 


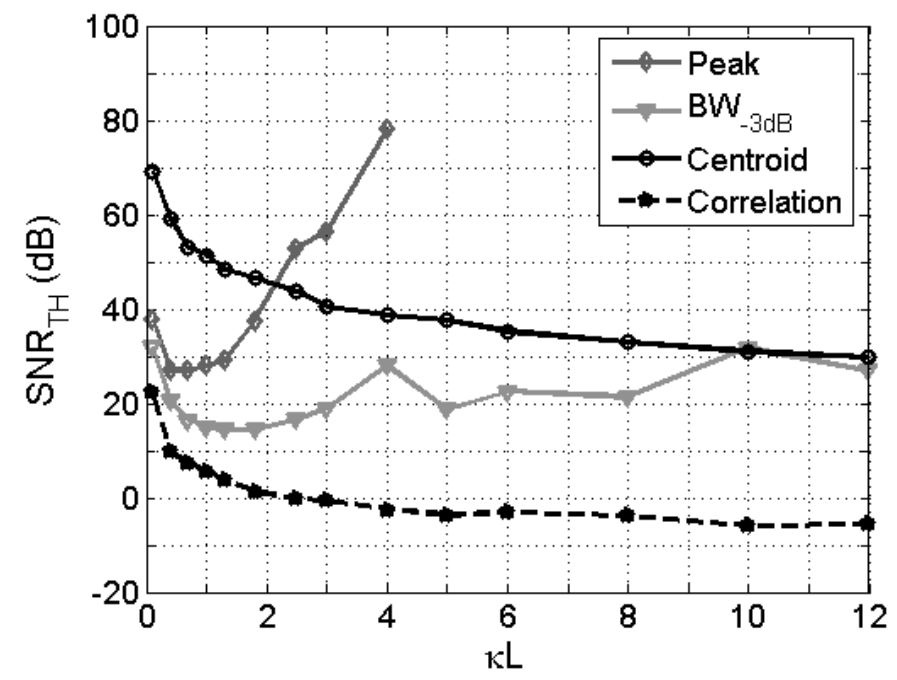

Fig. 5. Evaluation of the target SNR as a function of $\kappa \mathrm{L}$ for each tracking technique, performed with a 1000-sample Monte Carlo simulation with uniformly distributed noise.

The results shown in Figure 4-5 report, for the spectral correlation, the ideal case, based on the assumption that the reference spectra are available without noise. In order to evaluate the impact of the finite SNR of the initial spectrum, we repeated the simulation shown in Figure 4 adding white gaussian noise on both spectra. We report in Figure 6 the SNR penalty, i.e. the SNR variation from the ideal case, when the reference spectrum is measured with SNR equal to $30 \mathrm{~dB}$ and $20 \mathrm{~dB}$. In the first case, the penalty lays on a floor $(\sim 1.3 \mathrm{~dB})$ for $\kappa L>4$, and increases up to $8.0 \mathrm{~dB}$ for $\kappa L=1$; in the second case, the SNR penalty exhibits the same trend, but the floor value is $\sim 4.0 \mathrm{~dB}$ for $\kappa L>5$, with a maximum penalty of $14.9 \mathrm{~dB}$ for $\kappa L=1$. As expected, the penalty depends on the FBG bandwidth, since larger spectra are more tolerant to noise; again, this suggests than the application of spectral correlation provides better performances when applied to large FBGs. However, even supposing an inaccurate measure of the reference spectrum and a highly selective grating, the correlation technique is still the most advantageous in terms of $S N R_{T H}$. We also performed a further simulation in order to evaluate the effect of the laser linewidth on the spectral estimate: this was implemented by filtering the spectra with a sliding gaussian window with different width. No clear impact on the SNR has been noticed for $\kappa L>0.5$, even for a laser linewidth of $100 \mathrm{MHz}$; although a large laser spectral width tends to flatten the spectral profile, the correlation techniques is capable of dealing with noise fluctuations much greater than the effect of this spectral flattening.

To summarize, the Monte Carlo analysis clearly demonstrates the effect of FBG design on the peak tracking performance: strong large-bandwidth FBGs are preferred for correlation technique, while traditional techniques work better with narrow-bandwidth FBGs. This is an important consideration for practical FBG applications, as the majority of the research papers and industrial installations mount narrow-bandwidth FBGs, with $\kappa L=0.9-1.5$. Our simulation shows instead that an approximate increase of power budget figure up to $20 \mathrm{~dB}$ is feasible, it entirely relies on FBG sensors profile and 
peak tracking software, without any modification of the optical architecture, and works with any spectral scanning technique like the vast majority of FBG commercial interrogators.

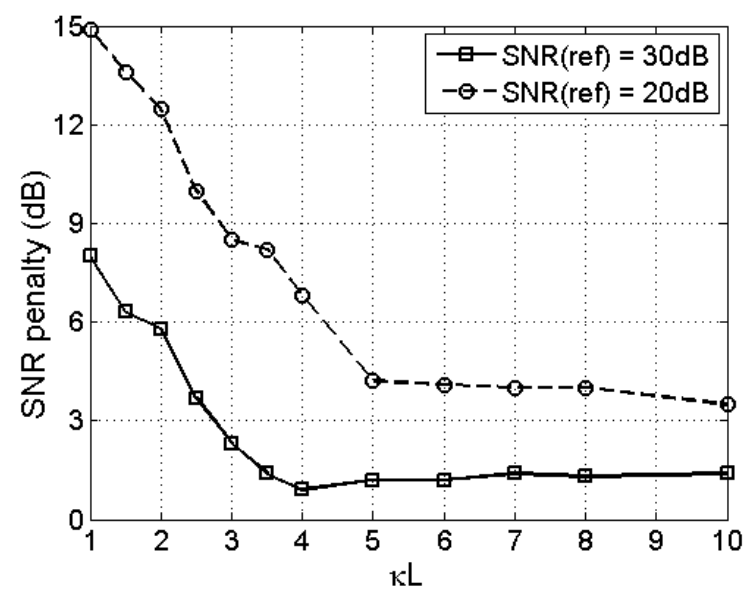

Fig. 6. Estimated SNR penalty with respect to the ideal case, when the reference spectrum is measured with SNR equal to 20 $\mathrm{dB}$ and $30 \mathrm{~dB}$.

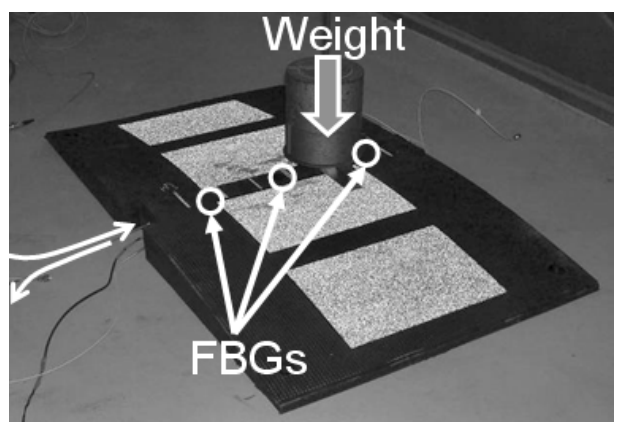

Fig. 7. Setup of the optical sensor for weight measurement on a road bump. Three FBGs, one for each side and one in the central part, are pre-strained and stuck on the back side of the bump. The system is loaded by applying a vertical static weight $(0-100 \mathrm{~kg})$ in each position.

\section{EXPERIMENTAL RESULTS}

The previously discussed peak tracking techniques have been applied in a practical case for the static characterization of a FBG-based weight sensor embedded in a road bump illustrated in Figure 7. Three FBGs have been mounted on the rear side of the dump in different positions; this arrangement permits to localize the load from different punctual strain measurements. The reflection spectra are recorded using a sweeping tunable laser (Photonetics Tunics-BT) and a power meter (HP8163A), over a wavelength grid with spacing of $10 \mathrm{pm}$. In order to load the structure, we vertically applied a weight of $0-100 \mathrm{~kg}$, with step $10 \mathrm{~kg}$, on the bump in different positions; hence, the spectral shift of each FBG depends on the weight applied to the sensor and on its position. As example, we report in Figure 8 the measured spectra of one edge-FBG when the load is applied exactly over the sensor. 


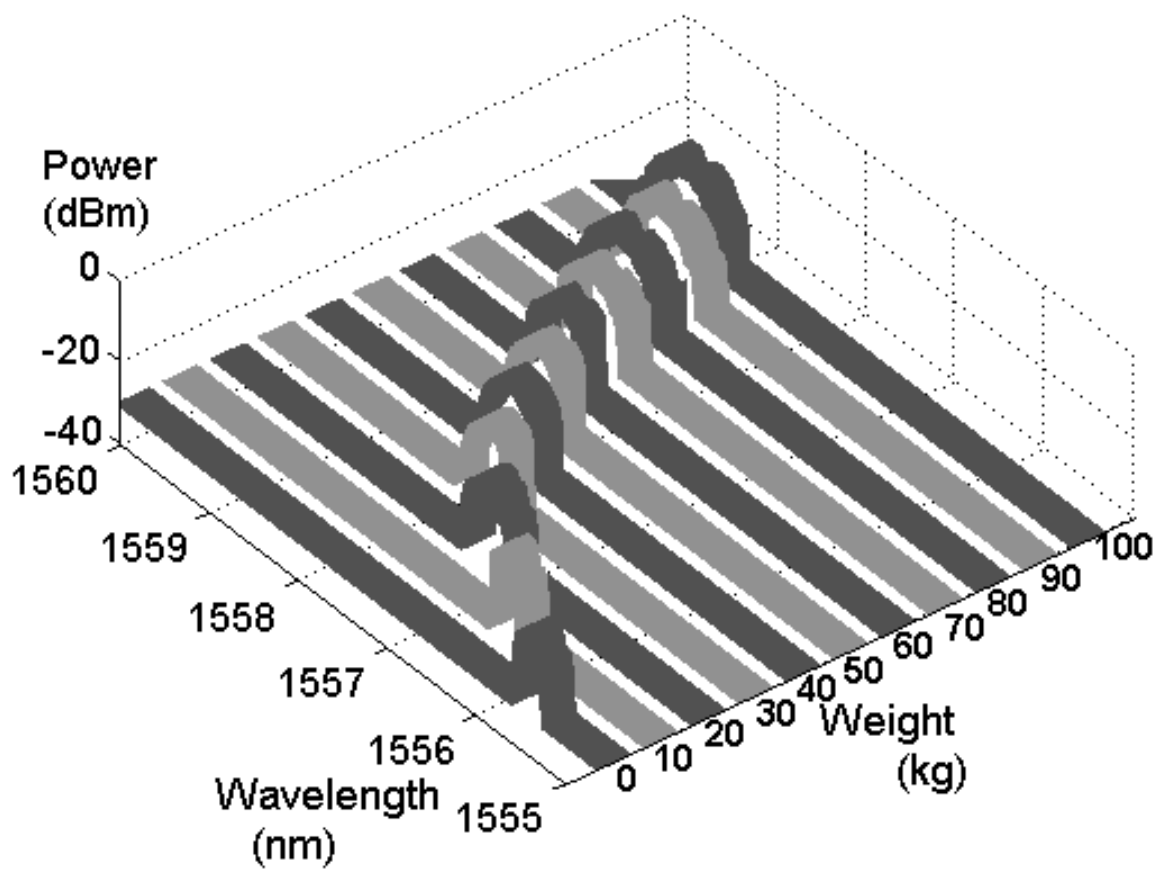

Fig. 8. Measured FBG spectrum when loaded by 0-100 kg weight vertically applied over the FBG.

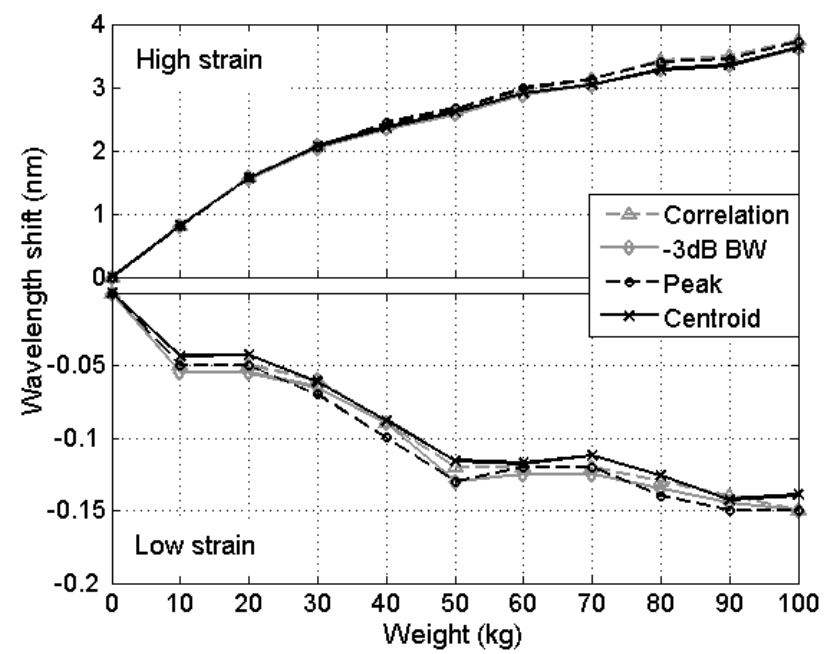

Fig. 9. Estimated wavelength shift as a function of weight, for all the applied peak tracking techniques; the high-strain curve refers to the FBG positioned under the load, while the low-strain curve refers to the FBG placed at the opposite side.

Figure 9 shows the wavelength shift, estimated with all the peak tracking techniques, as a function of the weight applied to the FBG placed under the load and to the one mounted at the opposite side; we defined as reference FBG spectrum the unloaded condition $(0 \mathrm{~kg})$. In the first case, the perturbation of the FBG is strong, and the wavelength shift exceeds the linear range even for weights higher than $30 \mathrm{~kg}$, hence the Bragg wavelength measurement is accurate only in the first part of the curve. Assuming the correlation technique as the correct estimate, the error on peak position with the other methods is $\pm 40 \mathrm{pm}$ for $10-20 \mathrm{~kg}$, hence a considerable uncertainty. As the strain increases, the deformed profile of the spectrum leads to an inaccurate estimate: spectral correlation and centroid 
techniques tend to find a sub-optimal alignment between the different spectra, while peak and BW tracking tend to follow the peak position of the deformed spectrum. The second FBG, instead, is weakly perturbed by the mechanical pressure, and exhibits a reduced spectral shift $(0.15 \mathrm{~nm})$. The spectral shift estimates lay in a range of $\pm 20 \mathrm{pm}$ with respect to the spectral correlation computation.

The application of a correlation technique can be exploited for determining whether the FBG wavelength shift exceeds the approximately linear region identified by (3). If that occurs, the FBG spectrum is significantly distorted, and the wavelength shift is no longer related to the mechanical/thermal excitation through a closed and repeatable relationship; consequently, the measure of the FBG peak is unreliable. Figures 8-9 show a typical example of this situation: due to the low elastic isolation between the bump surface and its rear side, the application of a heavy load produces an intense strain on the FBG, deforming its spectrum. A possible way for extracting this information is to evaluate the mean square error (MSE) between reference and measured spectra, after having found the optimum alignment:

$$
M S E=\frac{1}{N} \sum_{n=1}^{N}\left(S_{r e f}\left(\lambda_{n}\right) S_{m}\left(\lambda_{n}-\Delta \lambda_{S}\right)\right)^{2}
$$

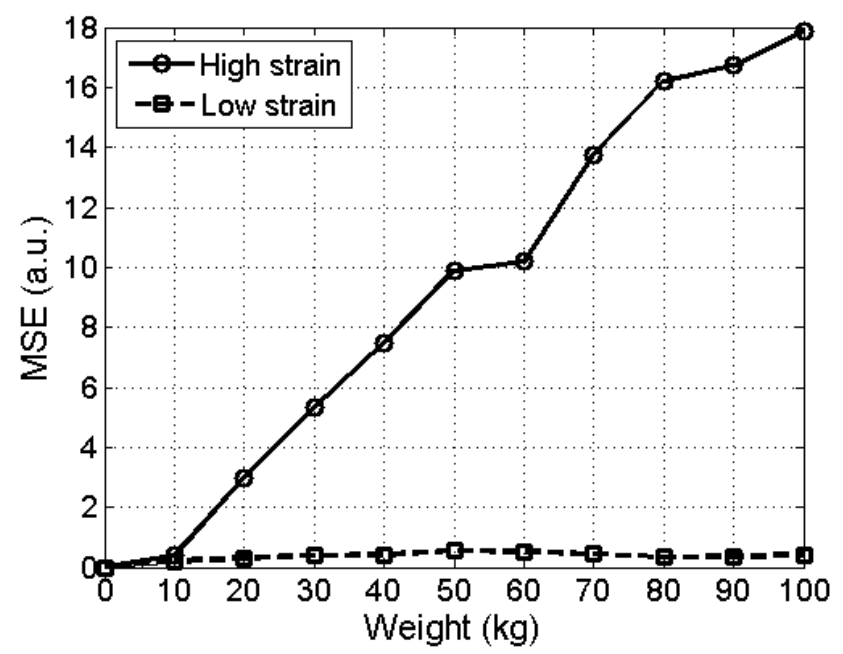

Fig. 10. The MSE of the spectral reconstruction is plotted versus the applied weight. The high-strain curve refers to the FBG positioned under the load, while the low-strain curve refers to the FBG placed at the opposite side.

This permits an immediate evaluation of the quality of the spectral reconstruction: as soon as the measured spectrum is distorted, the MSE grows with respect to the unstrained condition. We applied this technique to the measurement reported in Figures 8-9, in which we evaluated the MSE of the spectral alignment both for the FBG subjected to the heavy load and for the one positioned at the opposite side. The graph clearly shows that MSE achieved for the FBG far from the vertical load is approximately constant, confirming that the system operates in a linear regime. In contrast, for the FBG positioned close to the pressure point, the heavy load induces a perturbation that exceeds the linear regime even for light weight $(20 \mathrm{~kg})$, in which the MSE of the spectral reconstruction is approximately 8 times greater than the linear value. This result is compatible with Figure 8, in which 
we observe that the FBG spectrum for $20 \mathrm{~kg}$ weight is clearly much larger than the reference spectrum. We observe an almost linear increase of the MSE curve with respect to the applied weight; this suggests the possibility to exploit also the non-linear region of the strain/wavelength characteristic, expanding the FBG interrogation range.

\section{CONCLUSIONS}

The application of a spectral correlation-based technique to the peak estimation of a FBG permits a considerable increase of performances, expressed in terms of SNR resilience, of a typical interrogation system. Our statistical Monte Carlo-based simulations show that the gain, with respect to the standard wavelength tracking techniques, is about $20 \mathrm{~dB}$, depending on the $\kappa L$ parameter of the FBG: the employment of broad-band gratings permits to further improve the SNR tolerance. The increased noise tolerance achieved by this routine can be spent for enhancing the power budget of the FBG interrogator, increasing the maximum number of sensing FBGs as well as the acceptable losses over the fiber link, without changing the optical layout of the system. Finally, a practical case of the application of peak tracking methods has been discussed, pointing out the capability of the spectral correlation to detect whether the sensor exceeds the linear regime of the strain characteristic.

\section{REFERENCES}

[1] Y. J. Rao, “In-fibre Bragg grating sensors,” Meas. Sci. Technol., vol. 8, pp.355-375, 1997.

[2] A. D. Kersey, M. A. Davis. H. J. Patrick, M. LeBlane, K. P. Koo, C. G. Askins, M. A. Putnam, E. J. Fabriele, "Fiber grating sensors”, J. Lightw. Technol., vol. 15, pp. 1442-1463, 1997.

[3] E. J. Fabriele, "Fiber Bragg grating strain sensor: present and future in smart structures,” Opt. Photon. News, vol. 9, pp. 33-37, 1998.

[4] Y. Want, Y. Cui, B. Yun, “A fiber Bragg grating sensor system for simultaneously static and dynamic measurements with a wavelength-swept fiber lasers,” IEEE Photon. Technol. Lett., vol. 18, pp. 1539-1541, 2006.

[5] M. G. Xu, H. Geiger, J. L. Archambauldt, L. Reekie, J. P. Dakin, "Novel interrogation system for fibre Bragg grating sensors using an acousto-optic tunable filter,” Electron. Lett., vol. 29, pp. 1510-1511, 1993.

[6] M. A. Davis, A. D. Kersey, "Matched-filter interrogation technique for fibre Bragg grating arrays," Electron. Lett., vol. 31, n.10, pp. 822-823, 1995.

[7] R. W. Fallon, L. Zhang, A. Gloag, I. Bennion, "Identical broadband chirped grating-interrogation technique for temperature and strain sensing,” Electron. Lett., vol. 33, n. 8, pp. 705-707, 1997.

[8] Y. Yu, H. Zhao, “A novel demodulation scheme for fiber Bragg grating sensor systems,” IEEE Photon. Technol. Lett., vol. 17, n. 1, pp. 166-168, 2005.

[9] A. D. Kersey, T. A. Berkoff, W. W. Morey, "High resolution fibre grating based strain sensor with interferometric wavelength shift detection,” Electron. Lett., vol. 28, p. 236, 1992.

[10] S. T. Oh, W. T. Han, U. C. Paek, Y. Chung, "Discrimination of temperature and strain with a single FBG based on the birefringence effect,” Opt. Expr., vol. 12, n. 4, pp. 724-729, 2004.

[11] C.-C. Ye, S. E. Staines, S. W. James, R. P. Tatam, “A polarization-maintaining fibre Bragg grating interrogation system for multi-axis strain sensing,” Meas. Sci. Technol., vol. 13, pp. 1446-1449, 2002.

[12] A. Mendez, “Fiber Bragg grating sensors: a market overview,” Proc. SPIE, vol. 6619, pp. 661905.1-661905.6, 2007.

[13] T. Erdogan, “Fiber grating spectra,” J. Lightw. Technol., vol. 15, n. 8, pp. 1277-1294, 1997.

[14] A. Othonos, K. Kalli, Fiber Bragg gratings: fundamentals and applications in telecommunications and sensing, Artech House: Norwood, 1999.

[15] G. Meltz, W.W. Morey, "Bragg grating formation and germanosilicate fiber photosensitivity.” Proc. SPIE, vol. 1516, pp. 185-199, 1991.

[16] J. M. Gong, C. C. Chan, W. Jin, J. M. K. MacAlpine, M. Zhang, Y. B. Liao, "Enhancement of wavelength detection accuracy in fiber Bragg grating sensors using a spectrum correlation technique,” Opt. Comm., vol. 212, pp. 29-33, 2002.

[17] L. Negri, A. Nied, H. Kalinowski, A. Paterno, "Benchmark for peak detection algorithms in fiber Bragg grating interrogation and a new neural network for its performance improvement,” Sensors, vol. 11, n. 4 pp. 3466-3482, 2011.

[18] S. D. Dyer, P. A. Williams, R. J. Espejo, J. D. Kofler, S. M. Etzel, "Fundamental limits in fiber Bragg grating peak wavelength measurements," Proc. SPIE, vol. 5855, 2005.

[19] M. H. Kalos, P. A. Whitlock, Monte Carlo Methods, John Wiley \& Sons, 1986.

[20] Micron Optics, www.micronoptics.com.

[21] BaySpec, Inc., www.bayspec.com. 